\title{
Silibinin Loaded Solid Lipid Nanoparticles: Effect of Different Lipids and Surfactants on Physicochemical Properties of Nanoparticle
}

\author{
Priya Patel ${ }^{1}$, Mihir Raval ${ }^{1, *}$, Navin Sheth ${ }^{2}$ \\ 'Department of Pharmaceutical Sciences, Saurashtra University, Rajkot, Gujarat, INDIA. \\ ${ }^{2}$ Gujarat Technological University, Ahmedabad, Gujarat, INDIA.
}

\begin{abstract}
Background: Solid lipid nanoparticles are rapidly developing field of nanotechnology with several potential applications in drug delivery with spherical shape and an average diameter between 1 and $1000 \mathrm{~nm}$. Due to their size and its properties, solid lipid nanoparticles as drug delivery carrier have shown promising potential. Silibinin is a flavonoid with anticancer activity also possesses diverse pharmacologic effects. Methods: Solid lipid nanoparticles of silibinin are prepared by using lipids (Dynasan 114, Glyceryl mono stearate and Compritol 888ATO) with stabilizers (Soy lecithin, Poloxamer 407 and Tween 80). The prepared formulations have been evaluated for Particle size analysis, entrapment efficiency, in-vitro drug release, scanning electron spectroscopy, Fourier transform-infrared studies, Differential scanning calorimetric, $X$ - ray diffraction and Stability study. Results: EE raised with increasing the concentration of surfactants and shows inverse relationship for particle size. The in vitro release profiles of different formulations were studied and showed that sustained release of drug from all formulations. From fitting these data to various statistical models, the Korsmeyer Peppas model was proposed as the best model
\end{abstract}

to describe the statistical indices and mechanism of drug release. DSC and FTIR Shows no interaction between drug and excipients. By SEM Study its shows particles are spherical in shape. Stability studies revealed no prominent changes are seen upon storage. Conclusion: Formulation containing Compritol 888 ATO and Tween 80 (3\%) showed prolonged drug release upto $12 \mathrm{hrs}$, with smaller particle size and narrow particle size distribution, as compared to other formulations with different surfactants and lipids.

Key words: Solid lipid nanoparticle, Silibinin, Lipid matrix, Surfactant, Encapsulation efficiency.

\section{Correspondence}

Dr. Mihir Raval

Department of Pharmaceutical Sciences, Saurashtra University, Rajkot- 360005, Gujarat, INDIA.

Phone no: +91 9712727172

Email:mkraval@sauuni.ac.in

DOI: 10.5330/ijpi.2020.3.59

\section{INTRODUCTION}

As compare to conventional carrier system such as Polymeric nanoparticles, Liposomes, Microparticles, Solid lipid nanoparticles (SLN) is analternative carrier system. ${ }^{1}$ Nanoparticles made from solid lipids are attracting major attention as novel colloidal drug carrier as they have been proposed as an alternative particulate carrier system. ${ }^{2}$ Solid lipid nanoparticles are in submicron size range between $50-1000 \mathrm{~nm}$ and composed of lipids which are remain solid state at room temperature. Main goal for the preparation of SLN are increased drug stability, controlled drug release, Avoidance of organic solvent, Incorporation of lipophilic and hydrophilic drugs, improved bioavailability and no biotoxicity to the carrier. ${ }^{3-5}$ Silibinin $\left(\mathrm{C}_{25} \mathrm{H}_{22} \mathrm{O}_{10}\right)$, is a natural antioxidant, extracted from the medicinal plant silybum marianum and have been conventionally used for the treatment of cancer. ${ }^{6}$ Silymarin is the composed of several flavanolignans including Silibinin and its stereoisomers, namely silychristin, isosilychristin, isosilybin A, isosilybin B and silydianin. ${ }^{7}$ Silibinin has widely used as an antioxidant, hepatoprotective, anticancer, immunostimulatory, cell cycle arrest along with induction of apoptosis in different cancer cell lines. ${ }^{8-10}$ Some of the reported in vitro and in vivo study of Silibinin shown that they can effect on cancer in different three stages including progression, angiogenesis and tumour growth. ${ }^{11}$ The aim of the present research work is to study the effect of different types of Lipids matrix, types of Surfactants on the formulation and Stability of Solid Lipid Nanoparticles by characterizing SLN for Particle Size,
Polydispersity Index, Zeta Potential, Encapsulation Efficiency and Drug release.

Mehrdad Hamidi et al. studied the effect of surfactants on Physici chemical properties of repaglinide loaded solid lipid nanoaprticles. They used Stearic acid and glyceryl mono stearate (GMS) as lipid and phosphatidylcholin, Tween80, Pluronic F127, poly vinyl alcohol (PVA) and polyvinyl pyrrolidone (PVP as surfactant. They found that Tween 80 based SLNs showed smallest particle size and phosphatidylcholin-based SLNs indicated most prolonged drug release time along with highest drug loading capacity. Author conclude that mixture of phosphatidylcholin and Pluronic F127 was selected as the best surfactant to coat the lipid core containing stearic acid and glyceryl mono stearate. ${ }^{12}$

\section{MATERIALS AND METHODS}

Silibinin was purchased from Sigma Aldrich Pvt Ltd. Mumbai, Glycerol mono stearate, Dynasan 114, Compritol 888 ATO were kindly gifted by Gattefosse Pvt. Ltd., France as a lipids. Surfactants like Soy lecithin, Poloxamer 407 and Tween 80 were purchased from Sigma Aldrich Pvt. Ltd., Mumbai. All the reagents used were of analytical grade.

\section{Preparation of solid lipid nanoparticles by solvent emulsification-diffusion technique}

Silibinin loaded SLNs were prepared by a solvent emulsification diffusion technique using different lipids matrix (Dynasan 114, Glyceryl 
mono stearate and Compritol 888ATO) and (Soy lecithin, Poloxamer 407 and Tween 80 ) as surfactant. Lipid was dissolved in $2 \mathrm{ml}$ of ethanol: choloroform mixture (1:1) followed by dispersion of Silibinin. $5 \mathrm{ml}$ of aqueous surfactant solution was added drop wise in above solution under continuous stirring. Dispersion was homogenized by high speed homogenizer (IKA T25 Digital S22 Homogenizer, India) at 5000 RPM for $20 \mathrm{~min}$. Ice cold water was added to make up $50 \mathrm{ml}$ of volume and continuous stirring for $2 \mathrm{hr}$, for the diffusion of organic solvent into aqueous phase. Dispersion was centrifuged for $30 \mathrm{~min}$ at $5000 \mathrm{rpm}$ (Remi Laboratory Centrifuge,R-4CDX)for the separation of solid lipid nanoparticles. The filtrate was then freeze-dried in a lyophilizer (Delvac Pumps Pvt. Ltd., India).For the lyophilisation process, $1.5 \mathrm{ml}$ of nanoparticles suspension was poured into semi-stoppered glass vials with slotted rubber closures and freeze-dried at $-40^{\circ} \mathrm{C}$ for $72 \mathrm{~h}$. 5-10\% mannitol was added as cryoprotectants. The chamber pressure was maintained at $0.01 \mathrm{mbar}$ and the process was controlled by (Scanlaf CTS16a02) software. Nanoparticles were collected, kept in glass vials and stored in desiccator. ${ }^{13}$ The different formulation compositions were shown in Table 1.

\section{Evaluation of Prepared Nanoparticles Production yield}

The production yield was calculated as the total weight of the final product after drying, with respect to the initial total amount of drug along with ingredients used for the preparations of formulation. ${ }^{14}$ Production yield was calculated by using equation 1 .

$\%$ Production yield $=\frac{\mathrm{W} 1}{\mathrm{~W} 2} \times 100 \ldots \ldots \ldots . .(1)$

Where, W2 = Weight of starting materials

\section{Measurement of particle size, zeta potential and Polydispersity index}

Particle size, zeta potential and PDI value of nanoparticle were determined using Zetatrac (Microtrac Inc., USA) by light scattering approach. Nanoparticles were re-dispersed into de-ionized water. Put the sample into the sample holder for measurement of particle size, PDI and zeta potential.

\section{Measurement of Drug Entrapment Efficiency (\%EE)}

After centrifugation, supernant was collected and diluted with $10 \mathrm{ml}$ with $7.4 \mathrm{pH}$ buffer solution. This solution was analysed by UV-Visible Spectrophotometer at $288 \mathrm{~nm}$ using $\mathrm{pH}$ 7.4. It express the amount of un-

\begin{tabular}{|c|c|c|c|c|c|c|}
\hline Formulations & Lipid & $\% w / v$ & Surfactant & $\% w / v$ & Particle Size (nm) & Entrapment Efficiceny (\%) \\
\hline $\mathrm{F} 1$ & Dynasan 114 & 3 & Soylecithin & 1 & $1124 \pm 0.55$ & $56.32 \pm 1.25$ \\
\hline $\mathrm{F} 2$ & Dynasan 114 & 3 & Soylecithin & 2 & $987 \pm 1.21$ & $59.65 \pm 0.89$ \\
\hline F3 & Dynasan 114 & 3 & Soylecithin & 3 & $1025 \pm 2.14$ & $61.25 \pm 2.32$ \\
\hline $\mathrm{F} 4$ & Dynasan 114 & 3 & Poloxamer 407 & 1 & $985 \pm 2.54$ & $58.21 \pm 2.14$ \\
\hline F5 & Dynasan 114 & 3 & Poloxamer 407 & 2 & $785 \pm 1.58$ & $60.32 \pm 1.58$ \\
\hline F6 & Dynasan 114 & 3 & Poloxamer 407 & 3 & $625 \pm 1.32$ & $65.65 \pm 1.62$ \\
\hline F7 & Dynasan 114 & 3 & Tween 80 & 1 & $854 \pm 2.54$ & $60.21 \pm 1.08$ \\
\hline F8 & Dynasan 114 & 3 & Tween 80 & 2 & $921 \pm 0.88$ & $62.32 \pm 077$ \\
\hline F9 & Dynasan 114 & 3 & Tween 80 & 3 & $952 \pm 1.54$ & $64.52 \pm 2.32$ \\
\hline F10 & Glyceryl mono stearate & 3 & Soylecithin & 1 & $658 \pm 2.65$ & $55.28 \pm 2.05$ \\
\hline F11 & Glyceryl mono stearate & 3 & Soylecithin & 2 & $847 \pm 2.44$ & $60.25 \pm 2.14$ \\
\hline F12 & Glyceryl mono stearate & 3 & Soylecithin & 3 & $704 \pm 1.87$ & $61.54 \pm 3.21$ \\
\hline F13 & Glyceryl mono stearate & 3 & Poloxamer 407 & 1 & $662 \pm 0.88$ & $74.52 \pm 0.55$ \\
\hline F14 & Glyceryl mono stearate & 3 & Poloxamer 407 & 2 & $547 \pm 3.21$ & $76.25 \pm 1.54$ \\
\hline F15 & Glyceryl mono stearate & 3 & Poloxamer 407 & 3 & $485 \pm 0.54$ & $78.52 \pm 3.65$ \\
\hline F16 & Glyceryl mono stearate & 3 & Tween 80 & 1 & $502 \pm 1.54$ & $66.25 \pm 2.11$ \\
\hline F17 & Glyceryl mono stearate & 3 & Tween 80 & 2 & $389 \pm 1.89$ & $68.21 \pm 0.87$ \\
\hline F18 & Glyceryl mono stearate & 3 & Tween 80 & 3 & $354 \pm 2.54$ & $70.21 \pm 1.54$ \\
\hline F19 & Compritol 888ATO & 3 & Soylecithin & 1 & $645 \pm 0.87$ & $52.58 \pm 2.65$ \\
\hline F20 & Compritol 888ATO & 3 & Soylecithin & 2 & $587 \pm 1.54$ & $53.25 \pm 3.54$ \\
\hline $\mathrm{F} 21$ & Compritol 888ATO & 3 & Soylecithin & 3 & $515 \pm 2.66$ & $60.25 \pm 2.14$ \\
\hline F22 & Compritol 888ATO & 3 & Poloxamer 407 & 1 & $488 \pm 1.87$ & $65.58 \pm 0.87$ \\
\hline F23 & Compritol 888ATO & 3 & Poloxamer 407 & 2 & $465 \pm 1.22$ & $69.85 \pm 1.45$ \\
\hline F24 & Compritol 888ATO & 3 & Poloxamer 407 & 3 & $423 \pm 1.55$ & $70.25 \pm 2.65$ \\
\hline F25 & Compritol 888ATO & 3 & Tween 80 & 1 & $352 \pm 2.65$ & $75.65 \pm 3.21$ \\
\hline F26 & Compritol 888ATO & 3 & Tween 80 & 2 & $287 \pm 3.52$ & $80.25 \pm 0.98$ \\
\hline F27 & Compritol 888ATO & 3 & Tween 80 & 3 & $254 \pm 0.88$ & $82.35 \pm 1.32$ \\
\hline
\end{tabular}


entrapped drug in the supernant. ${ }^{15}$ The Drug Entrapment Efficiency was calculated by following equation 2

$\%$ Entrapment efficiency $=\frac{\mathrm{Da}+\mathrm{Ds}}{\mathrm{Da}} \times 100 \ldots \ldots \ldots . .(2)$

Where,

$\mathrm{Da}=$ Amount of drug added into formulation

Ds= Amount of un-entrapped drug

\section{In-vitro drug release study}

In-vitro drug release of the nanoparticle was performed by the dialysis bag diffusion technique. Nanoparticle equivalent to $50 \mathrm{mg}$ of Silibinin were filled into the dialysis bag and immersed in a dissolution chamber containing $300 \mathrm{ml}$ of $7.4 \mathrm{pH}$ phosphate buffer solution under continuous stirring at $100 \mathrm{rpm}$ at $37 \pm 0.5^{\circ} \mathrm{C}$. At predetermined time intervals, $2 \mathrm{ml}$ sample was withdrawn and scan at $288 \mathrm{~nm}$ by UV-Visible Spectrophotometer. In vitro drug release were further analysed by using model dependent approach. Various kinetic models like zero order, first order, higuchi, Hixson Crowell and webul model was applied to study the drug release mechanism from nanoparticle. ${ }^{16}$

\section{Characterization of Nanoparticles}

\section{Fourier Transform Infrared Spectroscopy}

FTIR analysis of Silibinin, Compritol 888 ATO, Tween 80, Physical mixture and Formulations were carried out for qualitative analysis. The pellet was kept in a sample holder of FTIR spectrophotometer (FTIR, Agilent CARY 630) and scanned in the range of $400-4000 \mathrm{~cm}-1$ at a resolution of $4 \mathrm{~cm}^{-1} .^{17}$

\section{Differential Scanning Calorimetry}

Differential scanning calorimeter measurements of Silibinin, Compritol 888 ATO, Tween 80 and Formulations were carried out with a differential scanning calorimeter (DSC-60, TA 6OWS, Shimadzu) under nitrogen flow. The DSC was calibrated for temperature and enthalpy measurements in the standard way, using the melting of pure indium metal, as reference material. DSC runs were conducted over a temperature range of 35 to $250^{\circ} \mathrm{C}$ at $10^{\circ} \mathrm{C} / \mathrm{min}$ under nitrogen flow rate of $100 \mathrm{ml} / \mathrm{min} .{ }^{17}$

\section{Shape and surface morphology}

Scanning electron microscopy was used for visualized the shape and surface morphology of the SLN. $10 \mathrm{mg}$ dried sample was loaded into the stub of scanning electron microscopy (SEM XL 30 ESEM). The stub was fixed into a sample holder and placed in vacuum chamber of scanning electron microscope. The SLNs were observed for surface characteristics.

\section{X-ray diffraction (XRD)}

Powder X-ray diffraction analysis was performed to verify new solid state formation. X-ray diffractogram of pure drug and silibinin loaded SLNs were collected in transmission using X-ray diffractometer. (Siemen's D-5000 Germany). XRD studies were performed on the pure silibinin and Silibinin loaded SLNs by exposed them to CuKa radiation $(40 \mathrm{kV}$, $30 \mathrm{~mA}$ ) and scanned from $2^{\circ}$ to $60^{\circ}, 2 \theta$ at a step size of $0.02^{\circ}$ and step time of 1 sec. $^{17}$

\section{Stability studies}

Stability studies of optimized formulations were carried out for 30 days by storing the formulations at two different temperatures, $4^{\circ} \mathrm{C}$ and $25 \pm$ $2^{\circ} \mathrm{C}$. Every 15 days interval formulations were evaluated for Particle size, Entrapment efficiency and drug content to determine any changes in the formulations. ${ }^{18}$

\section{RESULTS}

\section{Particle size analysis}

Table 1 shows the effect of the lipid and surfactant concentration on the particle size distribution of SLN prepared by using Dynasan 114, GMS and Compritol ATO 888 with Poloxamer 407, Tween 80 and Soy lecithin. The particle size ranged from $254 \mathrm{~nm}-1124 \mathrm{~nm}$. There was shown a significant difference in the particle size of SLN, with change in the lipids and surfactants.

\section{Effect of lipids on the particle size}

Silibinin loaded solid lipid nanoparticles, prepared by using different lipid matrix. Larger particle size was observed in SLN prepared by using Glyceryl mono stearate and Dynasan 114. This effect could be related to the melting point of the lipid, as both lipids having a higher melting point, resulting in slower lipid crystallization due to the hot homogenized state resulting in an increase in particle size. Along with Dynasan 114 dispersions remain liquid while kept at room temperature, when cooled down to $4^{\circ} \mathrm{C}$ they solidify. ${ }^{19}$ So it may be shows crystallinity during storage on the degradation, within 2-3 days after production, Dynasan 114 SLN do not stabilized with surfactants. The use of spatially specific lipids leads to greater distances between the glyceride fatty acid chains and general imperfections in the crystal and thus provides more space for molecules to fit and got larger particle size. SLN are degraded rapidly and completely after $120 \mathrm{~min}$ of degradation in the lipase solution. These degradation behaviour might be effect on particle size distribution. Decreasing Order of lipid Compritol 888 ATO > GMS > Dynasan 114

\section{Effect of surfactants on the particle size}

As the surfactant concentration increased particle size was decreased respect to all surfactants. SLN prepared using Soy lecithin as stabilizer showed larger particle size than the other surfactants irrespective of the lipids studied. Based on all respective results batch containing Compritol ATO 888 along with Tween 80 (3\%) shows the less particle size as compare to others. (Figure 1)

\section{Entrapment efficiency}

Entrapment efficiency is an important parameter for characterizing solid lipid nanoparticles. Factors such as type and concentrations of lipid and surfactants was play a vital role to attain optimal encapsulation efficiency. The entrapment efficiency of all the prepared SLN formulations is shown in Table 1. The entrapment efficiency of the SLN dispersions was found to be in the range of 50.21 to $82.35 \%$.

\section{Effect of surfactants on entrapment efficiency}

All SLN formulations prepared with a highest amount of surfactant such as $3 \%$ showed higher entrapment efficiency. This showed that the increase in the concentration of the surfactant increased the entrapment efficiency. Its might be due to the increase in the solubility of the drug in the lipid on increasing the concentration of the surfactant. This result was in agreement with the results obtained by Abdelbary et al. ${ }^{20}$ Formulations containing soy lecithin as surfactants showed lower entrapment efficiency compared to the other surfactants; this could be due to the lower HLB value of soy lecithin. Hence the entrapment efficiency of various SLNs stabilized with different surfactants and decreased in the order of tween $80>$ Poloxamer $407>$ soylecithin.

\section{In vitro drug release}

The in vitro release of silibinin from the SLN dispersion was found to be in the range of 50.21 to $81.40 \%$ at the end of $12 \mathrm{hr}$. 


\section{Effect of surfactants on in vitro drug release}

Formulations prepared by using GMS as a lipid matrix, with Tween 80 (3\%), Poloxamer $188(3 \%)$ and soy lecithin(3\%), as stabilizers showed a higher drug release from prepared Nanoparticle [82.32, 76.54 and $65.21 \%$, respectively]. This showed that the increase in the concentration of the surfactant there was an increase in the drug release from the SLN. However, this difference could not be attributed to the formulations [F3, F6 and F9] prepared by using Dynasan 114 as lipid matrix. These formulations showed a decrease in the drug release on the increase of the concentration of surfactant, which could be due to the lower melting point of the Dynasan 114. The results indicated that formulation [F25, F26, F27] Compritol ATO 888 along with Tween 80 exhibited a higher drug release. This could be due to the lower HLB value of Tween 80 than the other surfactants used as stabilizers. Thus the order of percentage of drug release was Tween $80>$ Poloxamer 407> Soy lecithin on the basis of the stabilizer.

\section{Influence of lipids on in vitro drug release}

Formulations containing compritol ATO 888 as a lipid matrix exhibited higher drug release than the formulations containing GMS as the lipid matrix, which showed a more sustained release. compritol ATO 888 produced less ordered crystals than GMS, leading to lower drug expulsion from the imperfect lattice, contributing to the prolonged release of the lipophilic drug such as silibinin. Compritol ATO 888 has a lower melting point as compare to GMS. The order of percentage of drug release was Compritol ATO 888> Glyceryl monostearate > Dynasan 114 on the basis of the lipid matrix. With Dynasan 114 its convert the instable lipid matrix after formulations, hence its shows the nonuniformity of release. Figure 2 shows the release profile of all the formulations containing the combination of Compritol ATO 888 with tween 80 . The study on drug release from solid lipid nanoparticles showed that drug release is influenced by lipid concentration. ${ }^{21}$

\section{Production Yield}

The production yield for prepared formulation was found in range between $34.58-65.14 \%$. Table 2 indicates that the \% yield was highest in batch $27(65.14 \%)$ while it was lower in batch $10(34.58 \%)$. This difference may be due to higher drug to lipid ratio or higher surfactant concentration.

\section{Polydispersibilty Index}

Polydispersibilty Index (PDI) shows size distribution of the formulation. PDI value $<1$ indicate narrow size distribution while in case of $>1$ indicate broad size distribution. Higher PDI indicates the high level of non-uniformity and its value was used to characterize the SLNs as monodisperse, homogeneous and heterogeneous system. PDI of all formulations were presented in Table 2. In this study PDI was found to be in range $0.15-0.89$.

\section{Zeta potential}

The zeta potential is an index of stability of SLN. Higher the zeta potential, higher the stability of formulation expected. Preferably zeta potential should be in range between $-30 \mathrm{mV}$ to $+30 \mathrm{mV}$ for moderate stability. The zeta potential of the prepared SLNs shown in Table 2. Zeta potential of all batches ranging between 21.21 to $34.56 \mathrm{mV}$ indicates more stability of formulation.

\section{Characterization of Nanoparticles}

\section{Fourier Transform Infra-Red Spectroscopy (FTIR)}

Fourier Transform Infra-Red Spectroscopy (FTIR) study was performed as per methodology. The FTIR spectra of SB, Compritol 888 ATO, Tween
80, Physical mixture and SLNs formulation spectra shown in Figure 3. FTIR spectrum of Silibinin showed characteristic peaks at 2858.11 $\mathrm{cm}^{-1}$ (-CH aromatic stretching), $1579.80 \mathrm{~cm}^{-1}$ (N-H bending), 1470.21 $\mathrm{cm}^{-1}$ ( $\mathrm{C}=\mathrm{C}$ stretching), $1368.76 \mathrm{~cm}^{-1}(\mathrm{O}-\mathrm{H}$ bending) were identified, Additionally in peak was appeared in the range $2200 \mathrm{~cm}^{-1}$ this was due to the broad band at $2000-2500 \mathrm{~cm}^{-1}$ relating to $\mathrm{O}-\mathrm{H}$ stretching vibration as well as a band at 2210 due to $\mathrm{C}-\mathrm{H}$ stretching. Which were same in SLN formulation. Thus, there was no any interaction between drug and excipients.

\section{Differential Scanning Calorimetry (DSC)}

Differential Scanning Calorimetry (DSC) study was performed as per methodology section. The DSC thermogram of pure SB showed sharp endothermic peak at $126.02^{\circ} \mathrm{C}$, corresponding to its melting point. DSC thermogram of Silibinin, Compritol 888 ATO, Tween 80 and formulation showed endothermic peak at $57.76^{\circ} \mathrm{C}, 168.75^{\circ} \mathrm{C}, 292.97^{\circ} \mathrm{C}$ respectively. DSC of the SLN formulation showed complete disappearance of characteristic SB peaks; the fact that the drug was molecularly distributed within the lipid matrix and after lyophilization there was no interaction between the drug and the excipient. The DSC curve of SB, compritol

Table 2: Production yield, PDI and Zeta potential for all batches of SLNs.

\begin{tabular}{cccc}
\hline Formulations & $\begin{array}{c}\text { Production yield } \\
\pm \text { SD }\end{array}$ & $\begin{array}{c}\text { PDI } \\
\pm \text { SD }\end{array}$ & $\begin{array}{c}\text { Zeta Potential } \\
\pm \text { SD }\end{array}$ \\
\hline F1 & $45.25 \pm 0.25$ & $0.89 \pm 2.58$ & $24.1 \pm 3.02$ \\
F2 & $47.52 \pm 1.21$ & $0.65 \pm 1.54$ & $27.34 \pm 1.58$ \\
F3 & $50.21 \pm 0.78$ & $0.42 \pm 0.65$ & $21.29 \pm 0.88$ \\
F4 & $52.32 \pm 0.98$ & $0.52 \pm 1.78$ & $32.15 \pm 2.58$ \\
F5 & $59.85 \pm 2.24$ & $0.32 \pm 2.54$ & $23.59 \pm 0.98$ \\
F6 & $62.32 \pm 2.65$ & $0.22 \pm 2.32$ & $22.56 \pm 0.85$ \\
F7 & $57.84 \pm 3.21$ & $0.63 \pm 2.65$ & $29.32 \pm 1.54$ \\
F8 & $54.65 \pm 0.98$ & $0.54 \pm 0.85$ & $30.34 \pm 2.54$ \\
F9 & $52.21 \pm 1.25$ & $0.24 \pm 0.66$ & $23.56 \pm 0.74$ \\
F10 & $34.58 \pm 2.32$ & $0.66 \pm 0.78$ & $21.21 \pm 1.65$ \\
F11 & $40.21 \pm 3.21$ & $0.60 \pm 2.14$ & $27.54 \pm 1.12$ \\
F12 & $43.21 \pm 1.45$ & $0.58 \pm 1.54$ & $29.87 \pm 1.98$ \\
F13 & $45.69 \pm 0.88$ & $0.45 \pm 1.69$ & $34.56 \pm 2.58$ \\
F14 & $52.12 \pm 1.54$ & $0.40 \pm 3.02$ & $22.89 \pm 0.65$ \\
F15 & $54.58 \pm 2.54$ & $0.35 \pm 1.54$ & $21.32 \pm 0.87$ \\
F16 & $52.32 \pm 1.65$ & $0.65 \pm 2.87$ & $27.56 \pm 1.05$ \\
F17 & $54.89 \pm 2.41$ & $0.54 \pm 1.45$ & $29.98 \pm 1.25$ \\
F18 & $59.65 \pm 0.88$ & $0.45 \pm 0.98$ & $30.25 \pm 0.98$ \\
F19 & $47.89 \pm 0.45$ & $0.65 \pm 0.54$ & $31.52 \pm 1.54$ \\
F20 & $49.65 \pm 1.54$ & $0.54 \pm 1.54$ & $24.78 \pm 2.32$ \\
F21 & $52.32 \pm 1.78$ & $0.41 \pm 2.65$ & $28.65 \pm 0.88$ \\
F22 & $63.25 \pm 2.54$ & $0.32 \pm 3.01$ & $32.54 \pm 1.45$ \\
F23 & $64.58 \pm 2.02$ & $0.22 \pm 0.78$ & $24.25 \pm 2.50$ \\
F24 & $58.65 \pm 1.56$ & $0.15 \pm 1.22$ & $26.89 \pm 1.22$ \\
F25 & $59.89 \pm 2.65$ & $0.25 \pm 2.55$ & $28.12 \pm 0.87$ \\
F26 & $62.32 \pm 1.58$ & $0.20 \pm 0.54$ & $30.54 \pm 1.65$ \\
F27 & $65.14 \pm 0.58$ & $0.18 \pm 0.98$ & $32.86 \pm 2.54$ \\
\hline
\end{tabular}


888 ATO, Tween 80 and SB loaded SLNs shown in Figure 4 A,B,C,D respectively.

\section{Scanning electron microscopy (SEM)}

The morphology of the optimized batch (F27) was examined by SEM. SEM photographs of the SB loaded SLN are shown in Figure 5. It can be seen that solid lipid nanoparticles are spherical in shape and having smooth surface.

\section{X-Ray Diffraction study (XRD)}

The X-ray diffraction spectra were recorded for pure SB and SB loaded SLNs shown in Figure 6 for investigating the crystallinity of the drug in solid lipid nanoparticles. Characteristic crystalline peaks of SB was observed at $2 \theta$ of $17.21,18.61,20.37,22.13,24.53$ and 72.55 indicating the presence of crystalline SB. SB loaded solid lipid nanoparticles showed very low intense peaks that indicates the major amount of $\mathrm{SB}$ was converted into amorphous form after lyophilisation.

\section{Stability studies}

No any major changes are seen in particle size and Drug content, But in entrapment efficiency of the solid lipid nanoparticles shown higher at $4^{\circ} \mathrm{C}$ as compared to solid lipid nanoparticles stored in $25^{\circ} \mathrm{C} \pm 2^{\circ} \mathrm{C}$. This may be due to more drug expulsion from the lipid matrices at higher temperature (Table 3 ). In the drug content after 15 days its shows $82.32 \pm 0.8$ and $79.32 \pm 1.1$ for $4^{\circ} \mathrm{C}$ and $25^{\circ} \mathrm{C}$ respectively, after 30 days for $25^{\circ} \mathrm{C}$ its shows $82.25 \pm 0.5$, its might be due to the slight aggregation shows on particle size and its shows the variation in drug content.

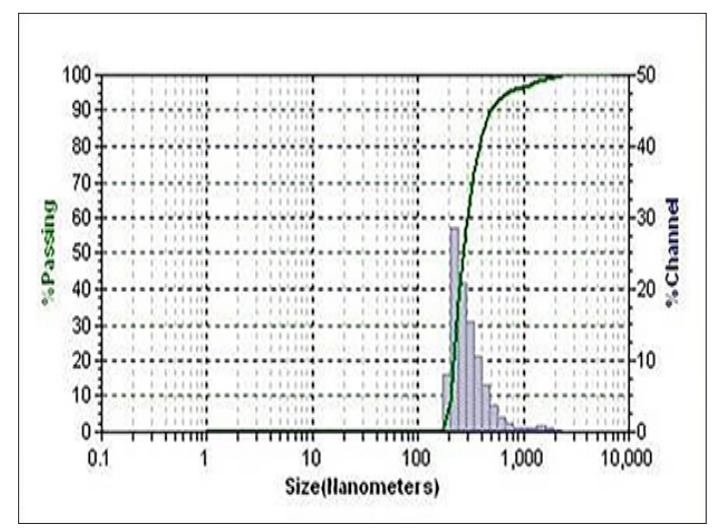

Figure 1: Particle size graph of optimized batch (F27).

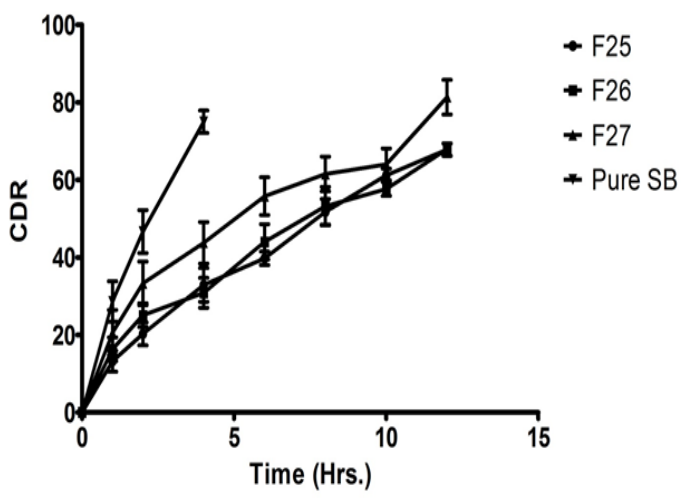

Figure 2: Release Profile of formulation.

All the values are mean \pm S.D $(n=3)$

\section{DISCUSSION}

SLNs were successfully prepared using Solvent emulsification diffusion technique. It's also offers advantages as compare to other method like easy to handle, simple and effective method, avoidance of organic solvents and heat during the preparation of solid lipid nanoparticles were the most important advantage of this technique. ${ }^{22}$ Compritol ATO 888 produced significantly smaller particles size than triglycerides that is GMS and Dynasan 114 at the same lipid concentration. This is reasonable since GMS and Dynasan 114 has higher MW and melting point than Compritol ATO 888. ${ }^{23}$ The fatty acid chain length had a significant effect on the particle size of SLNs and due to the higher melting point result in a more viscous dispersed phase that would be less homogenized effectively at the same conditions and in turn larger size. ${ }^{24}$ As the surfactant concentration increased particle size was decreased due to the reduce the surface tension. Due to the higher molecular weight of soy lecithin its shows larger particle size as compared to poloxamer 407 and tween $80 .{ }^{25}$ Preferably interspersed between the lipid layers while Tween 80 pile up at the outer surface of SLNs might hinder any particle agglomeration contributing to a decrease in particle size.

As the concentration of the surfactant increases at a constant amount of lipid, the surface of the formed SLNs is too small to adsorb all surfactant molecules which could lead to the formation of the drug's micellar solution. Therefore the drug's solubility was improved in water phase as well as reduce drug leaching due to the higher viscosity of the aqueous phase leading to a higher EE. ${ }^{26}$ In addition, the high content of hydroxyl groups in Tween 80 could result in greater intermolecular interaction via formation of hydrogen bonds between or inside molecules and hence, greater aqueous phase viscosity and coalescence of adjacent particles. ${ }^{27}$ On the other hand, increasing surfactant concentration above $2 \%$ can result in micellar solutions of the drug and lower EE. ${ }^{28}$ PDI value for optimized formulation was found to be 0.154 shows uniform particle size distribution. Prepared optimized formulation showed positively charged zeta potential indicating the presence of lipid present at surface of SLN and formulations remain stable. SEM study revealed that the particles were of spherical shape with smooth and nonporous surface. SB loaded solid lipid nanoparticles showed very low intense peaks that indicates the major amount of SB was converted into amorphous form after lyophilisation.

The amount of burst release of the drug from various formulations was substantially different. The amount of burst release has a direct connection to the amount of drug that existed on the nanoparticles surface. So it can be inferred that the greater the release of bursts, the greater the substance on the surfaces of the particles. To understand these differences in multiple SLNs, they seem to be linked to variations

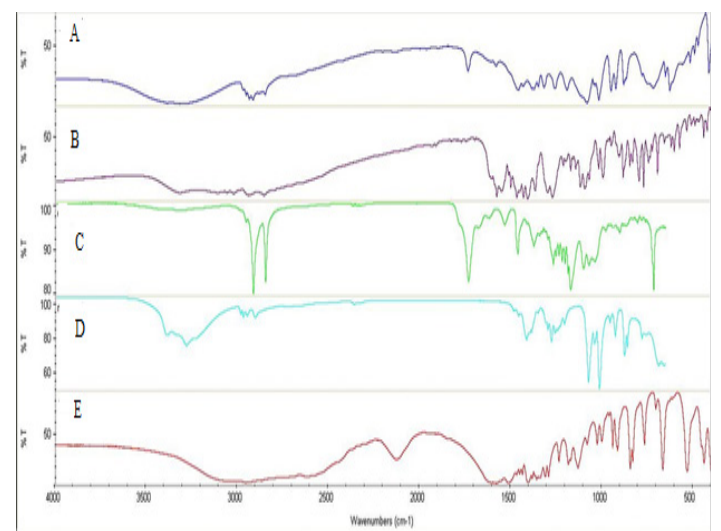

Figure 3: FTIR spectra of A) SB, B) Compritol 888 ATO, C) Tween 80, D) Physical mixture and E) SLNs formulation. 

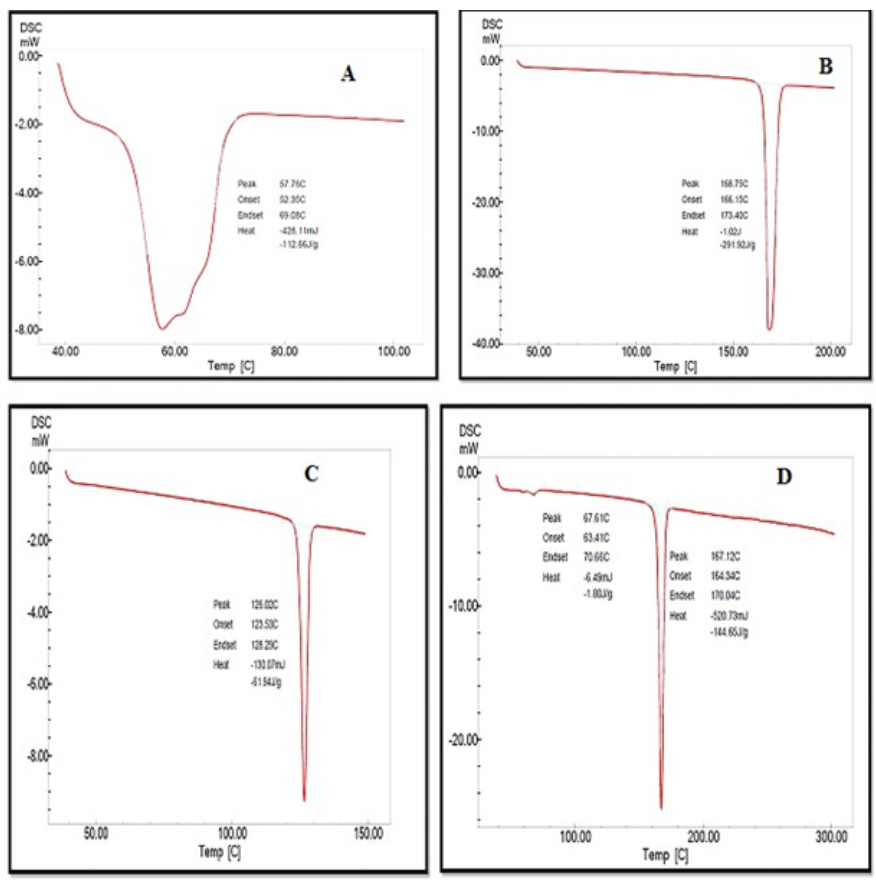

Figure 4: Figure 4: DSC curve of A) Silibinin, B) Compritol ATO 888, C) Tween 80 D) SB loaded SLN.

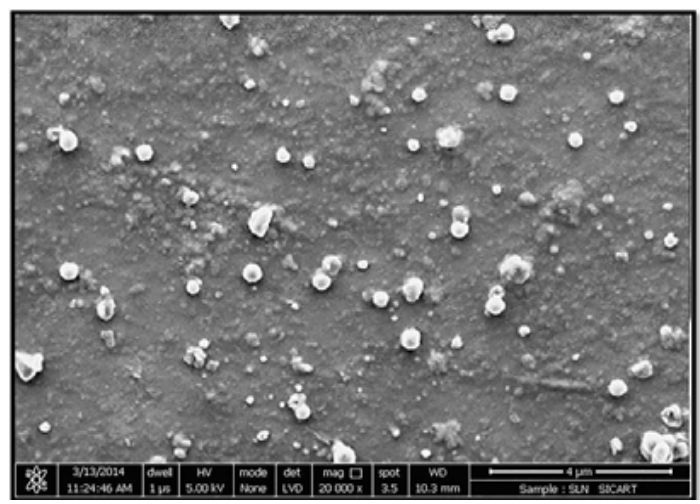

Figure 5: SEM of optimized formulation.

in particle sizes. The smaller ones contain larger particle surfaces and the smaller SLNs have shown greater burst release. In order to explore more, a correlation analysis was performed between these parameters and their corresponding particle sizes. As indicated there is a reverse correlation was seen between particle size and burst release $\left(R^{2}=0.985\right)$.

It suggested that the SB had a burst release in the first $2 \mathrm{~h}$ due to the presence of a small amount of free drug in the colloidal solution SB-SLN and the drug adsorbed on the nanoparticles surface and the cumulative release ratio at $2 \mathrm{~h}$ increased by up to $12 \mathrm{hr}$. Amount of drug released was much slower and controlled from the SLN dispersions prepared by using Compritol ATO 888 as the lipid matrix, with Tween $80(3 \%)$ as the surfactant, than that from the Silibinin-pure drug solution.

The in-vitro release of all the three batches of SLNs showed an interesting bi-phasic release with an initial burst effect. In the first hour, drug released was $18.76 \%, 14.72 \%$ and $12.8 \%$ for F25, F26 and F27 respectively. Afterwards, the drug release followed a steady pattern. The burst release in the first hour can be attributed to the drug loaded on the surface of SLNs. The same pattern of release is reported for curcumin ${ }^{29}$ and gemcitabine. $^{30}$ The values of in-vitro release were attempted to fit into various mathematical models like zero order, first order, Higuchi
Table 3: Stability Indicating Data.

\begin{tabular}{cccc}
\hline Optimized Batch & $\begin{array}{c}\text { Particle } \\
\text { Size }(\mathrm{nm})^{*}\end{array}$ & $\begin{array}{c}\text { Drug Content } \\
(\%)^{*}\end{array}$ & $\begin{array}{c}\text { Entrapment } \\
\text { efficiency (\%)* }\end{array}$ \\
\hline $\begin{array}{c}\text { Immediately after } \\
\text { preparation }\end{array}$ & $125 \pm 0.4$ & $85.42 \pm 0.3$ & $64.8 \pm 0.2$ \\
$15^{\text {th }}$ Day $4^{\circ} \mathrm{C}$ & $128 \pm 0.2$ & $82.32 \pm 0.8$ & $69.8 \pm 0.6$ \\
$15^{\text {th }}$ Day $25^{\circ} \mathrm{C}$ & $122 \pm 0.7$ & $79.32 \pm 1.1$ & $65.5 \pm 0.4$ \\
$30^{\text {th }}$ Day $4^{\circ} \mathrm{C}$ & $132 \pm 1.2$ & $83.25 \pm 0.6$ & $68.2 \pm 1.1$ \\
$30^{\text {th }}$ Day $25^{\circ} \mathrm{C}$ & $130 \pm 0.5$ & $82.25 \pm 0.5$ & $64.4 \pm 1.2$ \\
\hline
\end{tabular}

${ }^{*} n=3$

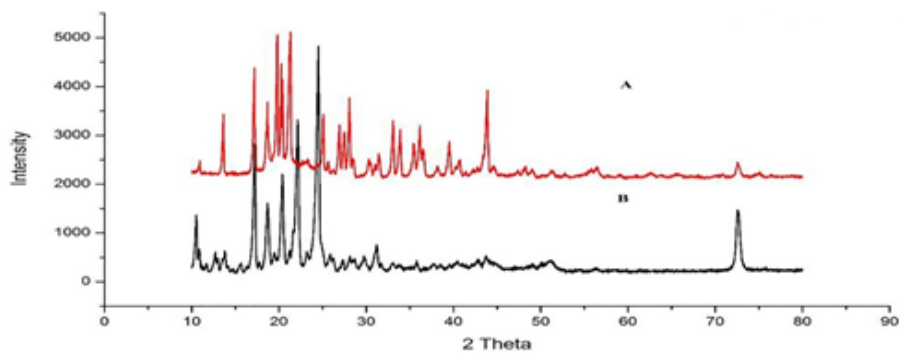

Figure 6: X-ray Diffraction spectra of pure SB (A) and SB loaded SLN (B).

matrix, Peppas and Hixson Crowell. Models with the highest correlation coefficient were judged to be the most appropriate model for the dissolution data. For formulations F25, F26 and F27 the best fitting linear parameter was that of Higuchi matrix with the ' $n$ ' value of $0.4269,0.4348$ and 0.4521 , respectively. This indicates that the drug release is controlled by diffusion of the drug through the pores. The results of stability testing revealed that there was no significant change in the particle size, Drug content and Entrapment efficiency.

\section{CONCLUSION}

It was observed that the lipid such as compritol ATO 888 and surfactant such as tween 80 by using Solvent emulsification diffusion method was a useful method for the successful incorporation of the low solubility drug such as silibinin. In fact, it could be assumed that the bioavailability could be improved if the particles in the nanometer range. It can therefore be concluded that solid lipid nanoparticles provide controlled release of the drug and enhance the bioavailability by nanoparticles of poorly watersoluble drugs as a drug delivery mechanism.

\section{ACKNOWLEDGEMENT}

Authors gratefully acknowledges to Gujarat council on science and technology (GUJCOST), Department of Science and Technology, Government of Gujarat, Gandhinagar for providing research grant to carry out this research work. Authors would like to acknowledge Gattefosse Pvt. Ltd., France for providing the lipids (Glycerol mono stearate, Dynasan 114, Compritol 888 ATO) as a gratis samples.

\section{CONFLICT OF INTEREST}

The authors declare no conflict of interest.

\section{ABBREVIATIONS}

DSC: Differential Scanning Calorimetry; FTIR: Fourier transform Infrared spectroscopy; GMS: Glyceryl Mono Stearate; SB: Silibinin; SEM: Scanning electron microscopy; SLN: Solid lipid nanoparticles. 


\section{REFERENCES}

1. S. Mukherjee S, Ray RS. Thakur, Solid lipid nanoparticles: A modern formulation approach in drug delivery system Ind. J Pharm Sci. 2009;714(4):349-58.

2. Jumaa M, Müller BW. Lipid emulsions as a novel system to reduce the haemolytic activity of lytic agents: Mechanism of the protective effect. Eur $J$ Pharm Sci. 2000;9(3):285-90.

3. Mozafari . Nanocarrier Technologies. Frontiers of Nano therapy, Springer. 2006;41-50.

4. Houli L, Xiaobin Z, Yukun M, Guangxi Z, Ling BL, Hong XL. Enhancement of gastrointestinal absorption of quercetin by solid lipid nanoparticles. J Cont Release. 2009;133(3):238-44.

5. Melike U, Gulgun Y. Importance of solid lipid nanoparticles (SLN) in various administration routes and future perspective. Int J Nanomedicine. 2007;2(3):289-300.

6. Cheung CW, Gibbons N, Johnson DW, Nicol DL. Silibinin-a promising new treatment for cancer. Anticancer Agents Med Chem. 2010;10(3):186-95.

7. Deep G, Singh RP, Agarwal C, Kroll DJ, Agarwal R. Silymarin and silibinin cause G1 and G2-M cell cycle arrest via distinct circuitries in human prostate cancer PC3 cells: A comparison of flavanone silibinin with flavanolignan mixture silymarin. Oncogene. 2006;25(7):1053-69.

8. Mateen S, Tyagi A, Agarwal C, Singh RP, Agarwal R. Silibinin inhibits human nonsmall cell lung cancer cell growth through cell-cycle arrest by modulating expression and function of key cell-cycle regulators. Mol Carcinog. 2010;49(9):47-58.

9. Nejati-Koshki K, Zarghami N, Pourhassan-Moghaddam M, Rahmati-Yamchi M, Mollazade $M$, Nasiri $M$, et al. Inhibition of leptin gene expression and secretion by silibinin: Possible role of estrogen receptors. Cytotechnology. 2012;64(6):719-26.

10. Surai PF. Silymarin as a natural antioxidant: An overview of the current evidence and perspectives. Antioxidants. 2015;4(1):204-47.

11. Liang $Z$, Yang $Y$, Wang $H, Y i$ W, Yan $X$, Yan J, et al. Inhibition of SIRT1 signalling sensitizes the antitumor activity of silybin against human lung adenocarcinoma cells in vitro and in vivo. Mol Cancer Ther. 2014;13(7):1860-72.

12. Hossein AE, Yousef J, Mehrdad H, Mohammad BJ. Repaglinide-loaded solid lipid nanoparticles: Effect of using different surfactants/stabilizers on physicochemical properties of nanoparticles. DARU Journal of Pharmaceutical Sciences. 2015;23(1):46.

13. Anand KK, Vuddanda PR, Priyanka K, Sanjay KS, Sanjay S. Development and Evaluation of Solid Lipid Nanoparticles of Raloxifene Hydrochloride for Enhanced Bioavailability. BioMed Research International. 2013;1-9.

14. Dinarvand R, Mirfattahi S, Atyabi F. Preparation, characterization and in vitro drug release of isosorbide dinitrate microspheres. J Microencapsul. 2002;19(1):7381.

15. Tayade PT, Kale RD. Encapsulation of water-insoluble drug by a cross-linking technique: Effect of process and formulation variables on encapsulation efficiency, particle size and in vitro dissolution rate. AAPS Pharm Sci Tech. 2004;6(1):112-9.

16. Bhardwaj, Burgess. A novel USP apparatus 4 based release testing method for dispersed systems. Int J Pharm. 2010;388(1-2):287-94.

17. Racault, Langlais F, Naslain R. Solid-state synthesis and characterization of the ternary phase Ti3SiC2. Journal of Materials Science. 1994;29(13):3384-92.

18. DelPozo-Rodríguez, Solinís, Gascón, Pedraz . Short- and long-term stability study of lyophilized solid lipid nanoparticles for gene therapy. European Journal of Pharmaceutics and Biopharmaceutics. 2009;71(2):181-9.

19. Bunjes $H$, Westesen $K$, Koch MHJ. Crystallization tendency and polymorphic transitions in triglyceride nanoparticles. Int J Pharm. 1996;129(1-2):159-73.

20. Abdelbary G, Fahmy RH. Diazepam - loaded solid lipid nanoparticles: Design and characterization. AAPS Pharm Sci Tech. 2009;10(1):211-9.

21. Naikwade SR, Bajaj AN, Gurav P, Gatne MM, Singh S. Development of budesonide microparticles using spray-drying technology for pulmonary administration: Design, characterization, in vitro evaluation and in vivo efficacy study. AAPS Pharm Sci Tech. 2009; 10(3):993-1012.

22. Nikam S, Chavan M, Sharma PH. Solid Lipid Nanoparticles: A Lipid Based Drug Delivery. Innov Pharm Pharmacother. 2014;2(3):365-76.

23. Triplett M, Rathman JF. Optimization of $\beta$-carotene loaded solid lipid nanoparticles preparation using a high shear homogenization technique. J Nanopart Res. 2009;11(3):601-14.

24. Jain $A$, Singh SK, Singh $Y$, Singh S. Development of lipid nanoparticles of diacerein, an antiosteoarthritic drug for enhancement in bioavailability and reduction in its side effects. J Biomed Nanotechnol. 2013;9(5):891-900.

25. Xie S, Zhu L, Dong Z, Wang X, Wang Y, Li X, et al. Preparation, characterization and pharmacokinetics of enrofloxacin-loaded solid lipid nanoparticles: Influences of fatty acids. Colloids Surf B: Biointerfaces. 2011;83(2):382-7.

26. Ravi P, Aditya N, Kathuria H, Malekar S, Vats R. Lipid nanoparticles for oral delivery of raloxifene: Optimization, stability, in vivo evaluation and uptake mechanism. Eur J Pharm Biopharm. 2014;87(1):114-24.

27. Singh S, Dobhal AK, Jain A, Pandit JK, Chakraborty S. Formulation and evaluation of solid lipid nanoparticles of a water soluble drug: Zidovudine. Chem Pharm Bull. 2010;58(5):650-5

28. Waheed MI, Alomrani AH, Eldeen AB. Novel sulpiride-loaded solid lipid nanoparticles with enhanced intestinal permeability. Int $\mathrm{J}$ Nanomedicine. 2014;9:129-44.

29. Tsai YM, Chien CF, Lina LC, Tsai TH. Curcumin and its nano formulation: the kinetics of tissue distribution and blood brain barrier penetration. Int $\mathrm{J}$ Pharm. 2011;416(1):331-8.

30. Fengqiang W, Yan W, Qing ZM, Yuan C, Bo Y. Development and characterization of folic acid-conjugated chitosan nanoparticles for targeted and controlled delivery of gemcitabinein lung cancer therapeutics. Artif Cells Nanomed Biotechnol. 2017:45(8):1530-8.

Article History: Submission Date : 10-04-2020; Revised Date : 03-05-2020; Acceptance Date : 23-07-2020

Cite this article: Patel P, Raval M, Sheth N . Silibinin Loaded Solid Lipid Nanoparticles: Effect of Different Lipids and Surfactants on Physicochemical Properties of Nanoparticle. Int. J. Pharm. Investigation, 2020;10(3):332-8 